\title{
Banco de Preguntas: una estrategia para el desarrollo lectoescritor en la educación superior
}

Alexander Tovar-Aguirre

orcid.org/oooo-0oo1-6983-9312

Pontificia Universidad Javeriana (Cali),

Colombia

alexander.tovar@javerianacali.edu.co

\section{Resumen}

Ante las limitaciones en lectoescritura que se reportan en estudiantes de educación superior, este artículo tiene como objetivo presentar los alcances del Banco de Preguntas, una herramienta diseñada para guiar de forma sistemática el desarrollo del lectoescritor competente en la universidad. Basado en un enfoque sociocultural del desarrollo psicológico, el Banco de Preguntas retoma una práctica cotidiana del salón de clase y la transforma en un dispositivo pedagógico que regula la construcción de competencias en lectura y escritura a través de diferentes niveles de complejidad. En este estudio, de carácter cualitativo descriptivo, se exploran los efectos subjetivos del Banco de Preguntas en la formación lectoescritora de 24 estudiantes universitarios. Al finalizar el semestre académico cada participante fue instruido a realizar una reflexión escrita sobre su experiencia personal con el uso de la herramienta. El análisis de contenido de estos documentos indica que el uso del Banco de Preguntas favorece la emergencia de un lector más activo, estimula el desarrollo de habilidades metacognitivas y consolida formas de relación profesor-estudiante dinámicas y democráticas en el aula.

\section{Palabras clave (fuente: tesauro de la UnEsco)}

Enseñanza superior; escritura; interrogación (método pedagógico); lectura; psicología del desarrollo.

\footnotetext{
Recepción: 14/03/2018 | Envío a pares: 18/04/2018 | Aceptación por pares: 07/03/2019 | Aprobación: 09/03/2019 


\title{
Question Bank: A Strategy for Literacy Development in Higher Education
}

\begin{abstract}
Given the literacy limitations that are reported in higher education students, this article aims to present the scope of the Question Bank, a tool designed to systematically guide the training of a competent writer-reader at university. Building on a sociocultural approach to psychological development, the Question Bank reintroduces a daily classroom practice and turns it into a pedagogical device that regulates the acquisition of reading and writing skills at different levels of complexity. In this qualitative descriptive study, the subjective effects of the Question Bank are explored in the literacy education of 24 university students. At the end of the semester each participant was instructed to write a reflection on their personal experience with the tool. Content analysis of these documents suggests that the use of the Question Bank favors the emergence of a more active reader, stimulates the learning of metacognitive skills, and consolidates dynamic and democratic teacher-student relations in the classroom.
\end{abstract}

\section{Keywords (Source: UnEsco Thesaurus)}

Higher education; writing; questioning (teaching method); reading; developmental psychology. 


\section{Banco de Perguntas: uma estratégia para o desenvolvimento da lectoescrita no ensino superior}

\section{Resumo}

Diante das limitações na lectoescrita, presentes em estudantes do ensino superior, este artigo tem como objetivo apresentar os alcances do Banco de Perguntas, uma ferramenta desenhada para guiar, de forma sistemática, o desenvolvimento da lectoescrita competente na universidade. A partir de uma abordagem sociocultural do desenvolvimento psicológico, o Banco de Perguntas retoma uma prática cotidiana da sala de aula e transforma em um dispositivo pedagógico que regula a construção de competências em leitura e escrita por meio de diferentes niveis de complexidade. Neste estudo, de caráter qualitativo descritivo, são explorados os efeitos subjetivos do Banco de Perguntas na formação da lectoescrita de 24 estudantes universitários. Ao finalizar o período acadêmico, cada participante foi instruído a realizar uma reflexão escrita sobre sua experiência pessoal com o uso da ferramenta. A análise de conteúdo desses documentos indica que o uso do Banco de Perguntas favorece a emergência de um leitor mais ativo, estimula o desenvolvimento de habilidades metacognitivas e consolida formas de relação professor-estudante dinâmicas e democráticas na sala de aula.

\section{Palavras-chave (Fonte: Tesauro da Unesco)}

Educação superior; escrita; interrogatório (método pedagógico); leitura; psicologia do desenvolvimento. 
La recurrencia, variedad y complejidad con las que aparecen las prácticas de lectura y escritura durante la formación pre y posgradual revelan no solo el lugar central que tiene la comprensión y producción textual como instrumentos para la enseñanza y el aprendizaje, sino una estrecha relación entre la competencia lectoescritora del estudiante y la calidad de la formación profesional. Se espera que esta competencia sea desarrollada en los niveles de educación básica y media, dejando al nivel superior el camino expedito para su uso en la profundización conceptual y teórica en las diferentes áreas del conocimiento.

En la práctica, esta expectativa se ve contrariada por las importantes limitaciones que los estudiantes presentan al enfrentar actividades cotidianas de lectoescritura académica (Ochoa y Aragón, 2007). Tradicionalmente dichas limitaciones se han explicado a partir de la formación escolar previa, el capital cultural o las habilidades cognitivas de los estudiantes; sin embargo, estas explicaciones, además de individualizar el problema y localizarlo en el estudiante, soslayan el análisis de las particulares condiciones y exigencias de producción sociodiscursiva que se privilegian en la universidad, así como las prácticas pedagógicas que permiten lidiar con tales exigencias (Ortiz, 2011; Carlino, 2001).

Transferir a la práctica pedagógica universitaria el problema de las competencias lectoescritoras de los estudiantes implica cuestionar las formas mediante las cuales los docentes contribuyen o no al desarrollo de dicha competencia (Carlino, 2001). Asumiendo ese desafío, este artículo presenta el impacto del Banco de Preguntas en la formación lectoescritora de un grupo de estudiantes universitarios, como estrategia pedagógica cuyo objetivo es promover un lectoescritor competente para la comprensión y producción textual dentro del género científico académico universitario. En su doble propósito de promover el éxito académico de los estudiantes y el fortalecimiento de las prácticas pedagógicas en lectoescritura, la estrategia resalta una vía posible y organizada para la enseñanza sistemática de esta competencia durante la formación universitaria.

Como preámbulo, se introducen algunas consideraciones sobre la lectoescritura en educación superior y la perspectiva teórica que fundamenta la propuesta. Al final del documento se discuten los resultados y las implicaciones del uso de esta herramienta para el mejoramiento de la calidad en la formación universitaria.

\section{El género "científico académico" en educación superior}

Más que un sistema de formas abstractas que cumplen una función representacional en la comunicación, el lenguaje es un fenómeno que surge y se transforma en las prácticas discursivas cotidianas de una comunidad lingüística específica. Según Bajtín (1997) son las distintas esferas de la actividad humana las que crean los diversos "géneros del discurso" (Faraco, 2003, p. 112) que imponen a sus hablantes los límites del qué y el cómo de la enunciación. En la esfera de la actividad humana universitaria, las condiciones de enunciación difieren de cualquier otra práctica social, incluso de aquellas realizadas en los niveles de educación básica y media (Olave, Cisneros y Rojas, 2013; Carlino, 2007); por lo tanto, no hay necesariamente una continuidad natural entre las habilidades lectoescritoras desarrolladas en estos últimos niveles y el nivel superior.

Lo anterior implica desnaturalizar la idea de que "saber" leer y escribir habilita automáticamente a los estudiantes para afrontar las demandas particulares de comprensión y producción textual de la educación superior (Estienne y Carlino, 2004), con lo que además se cuestiona la oferta curricular y metodológica que las instituciones universitarias ponen a disposición de los estudiantes para apropiarse del género científico académico y facilitar la formación profesional de calidad (Aguirre, 2015). Esta última consideración es particularmente relevante cuando se considera que, gracias a las políti- 
cas públicas de acceso democrático y permanencia en la universidad que promueve el gobierno colombiano (i.e. Ser Pilo Paga), grupos históricamente alejados de la formación profesional están accediendo a la educación superior. Estos grupos, cuya experiencia sociocultural con las formas elaboradas del lenguaje está disminuida por las condiciones socioeconómicas de su contexto familiar, serían susceptibles de experimentar mayores dificultades con el género científico académico en comparación con aquellos estudiantes que provienen de sectores sociales mejor acomodados (Ovejero, 2012a; Brunner, 2010).

Así, la lectoescritura en la universidad configura uno de los principales desafíos que enfrentan tanto los estudiantes de las diferentes clases sociales (Aguirre, 2015) como la propia institución educativa, toda vez que esta competencia se relaciona no solo con la calidad de la formación profesional sino también con el riesgo de deserción estudiantil (Olave et al., 2013). Frente a este desafío, ¿cuáles serían las posibles rutas de reflexión para abordar el problema? La propuesta que aquí se desarrollada se distancia de cualquier concepción individualista que localice las causas de la (in)competencia lectoescritora en las capacidades cognitivas, biológicas y familiares del estudiante y asume la responsabilidad por la formación de un lectoescritor competente para la comprensión y producción textual en la universidad.

Basado en un enfoque sociocultural del desarrollo psicológico, este trabajo entiende la lectoescritura en la educación superior como una forma compleja de pensamiento humano que se (trans)forma en las prácticas cotidianas académicas y que es "internalizada" (Rosas y Balmaceda, 2008, p. 39) por el estudiante a medida que interactúa con otros sujetos, con la mediación semiótica de los instrumentos culturales presentes en dichas actividades. Esta forma de abordar el problema se inscribe en lo que Ortiz denomina el "enfoque sociocognitivo" (2011, p. 24), una perspectiva que hace énfasis especial en el carácter situado de este tipo de competencia y en su importancia como objeto de enseñanza explícita y sistemática en la universidad (Ortiz, 2011, Carlino, 2007). En este sentido, el presente estudio aporta elementos de reflexión e intervención para un tema cuya historia en Colombia alcanza solo una década y dentro de un enfoque que atiende a la complejidad del contexto sociocultural y a sus instrumentos de mediación para comprender las "dificultades" académicas del individuo (Ortiz, 2011).

\section{La mediación semiótica para el lectoescritor universitario}

En los enfoques socioculturales del desarrollo humano destaca el papel fundamental de los instrumentos de mediación semiótica (Baquero, 2009), pues estos configuran las condiciones objetivas y subjetivas a partir de las cuales toma forma la psicología del individuo (Cubero, 2005). Referido a este estudio, lo anterior significa que el origen del lectoescritor competente no se ubica en una dimensión interna del sujeto, sino en una dimensión externa e intersubjetiva delimitada por el propio dispositivo pedagógico (Larrosa, 2000), en este caso denominado Banco de Preguntas, cuya actividad central es formular una pregunta-respuesta a partir del texto programado para una sesión de clase.

Para cumplir su propósito de ser instrumento de mediación semiótica que atiende al desarrollo sistemático de un lectoescritor que identifica ideas, conceptos y problemáticas centrales de los textos, analiza la evidencia y coherencia de sus argumentos y es capaz de trazar relaciones entre el texto y otras fuentes bibliográficas, el Banco de Preguntas ha sido pensado a partir de tres consideraciones. La primera, retomar una práctica central y cotidiana en la interacción profesor-estudiante (Collante, 2010) y restituir su valor para la promoción del diálogo y el desarrollo de una postura activa y ética frente al texto y su comprensión (Silvestri, 2006; Valera y Madriz, 2002). En este sentido, el Banco de Preguntas reivindica el cuestionamiento como forma de relación intersubjetiva activa que le permite al estudiante discutir con las diferentes voces que se recrean en 
una cadena discursiva de la que el texto es una de las múltiples entradas (Bajtín, 1997).

La segunda consideración implica el reconocimiento de que los estudiantes se desarrollan mientras aprenden y, por lo tanto, los instrumentos de aprendizaje deben tener la capacidad de adaptarse a estos avances para promover nuevos y más complejos estados del conocimiento. Este rasgo "extensivo" (Otálora, 2011) del Banco de Preguntas se refleja en cuatro niveles de complejidad creciente que inician en el diagnóstico y la familiarización con la herramienta. El primer nivel establece la que Vigotsky (1988, p. 133) denominó "zona de desarrollo real", a partir de la cual se pueden construir las diferentes "zonas de desarrollo próximo" que llevarían gradualmente al estudiante desde una lectoescritura superficial y periférica (nivel I) a comprender conceptos relevantes de los textos (nivel II) (Stone, 1999), para luego trabajar con ideas y argumentos centrales de los autores (nivel III) que deben tener presentes para conectar y discutir con los nuevos textos a trabajar (nivel IV). Con una mirada constructivista, cada uno de estos niveles retoma los anteriores y los reorganiza a la luz de las nuevas exigencias planteadas.

La tercera consideración refiere a la importancia de la interacción interindividual en la construcción y reconstrucción de los saberes. La denominación Banco de Preguntas responde a la idea de una "economía solidaria" dentro de la cual los estudiantes alimentan un "deposito" público de preguntas-respuestas que sirven tanto para la discusión en aula como para la formulación de actividades de evaluación escrita. En este sentido, los problemas que deben ser abordados en las evaluaciones escritas destacan la participación de los estudiantes en su propio proceso formativo-evaluativo y el de sus pares académicos.

Finalmente, se debe resaltar que el Banco de Preguntas es un instrumento que podría jugar un papel importante en el desarrollo de las habilidades metacognitivas, las cuales son un aspecto clave en el proceso de comprensión y producción textual (Ochoa y Aragón, 2007) y en el desarrollo de estrategias de aprendizaje autorregulado, del tipo "aprender a aprender" (Osses y Jaramillo, 2008; Silvestri, 2006). Dos características del instrumento aportan en dicho sentido. En primer lugar, el estudiante es al mismo tiempo el autor y el destinatario de la pregunta-respuesta; por consiguiente, se espera que conozca la respuesta para formular el cuestionamiento. Sin embargo, el proceso de formulación de una pregunta que coincida con la comprensión inicial que tiene el estudiante podría desencadenar un bucle de reorganizaciones sucesivas del conocimiento sobre el texto (Perinat, 1995), donde la nueva pregunta lleva a una nueva comprensión del texto, que, a su vez, conduce a una nueva reformulación de la pregunta, hasta percibir concordancia entre la comprensión y el cuestionamiento. Dado que esta concordancia no está en el texto, sino en el propio pensamiento que la ha promovido, la operación cognitiva se despliega sobre sí misma y genera procesos metacognitivos. En segundo lugar, si bien el estudiante es el primer destinatario de su preguntarespuesta, no es el último. Como se señaló respecto de la tercera consideración, el Banco de Preguntas es de uso y acceso público, con lo cual el estudiante no solo debe encontrar la concordancia entre la comprensión y el cuestionamiento, sino presuponer al posible lector, para ajustar las condiciones de la enunciación, es decir, crear una instancia del discurso en su pregunta-respuesta (el posible lector) que sirva como referente de revisión y ajuste de su producción textual. En conjunto, estas exigencias favorecen el rol activo del lector y la actividad intersubjetiva, en relación tanto con las ideas del texto como con los pares académicos y el docente, quienes conforman el primer público de la producción textual.

\section{Metodología}

El Banco de Preguntas se utilizó como estrategia pedagógica en dos cursos a cargo del autor del presente estudio en los semestres lectivos de $2016 \mathrm{y}$ 2017. Estos cursos, ubicados en el ciclo de profesiona- 
lización de la carrera de Psicología de una universidad privada de Cali (Colombia), estaban orientados a desarrollar competencias disciplinares y profesionales para la actuación del psicólogo en el área educativa; por lo tanto, las fuentes bibliográficas sobre las que se realizó el Banco de Preguntas son textos teóricos y teórico-metodológicos que tienen exigencias medias-altas de comprensión lectora para su adecuada apropiación.

Cada nivel de complejidad del Banco de Preguntas se trabajó durante dos o tres sesiones de clase (un texto por sesión). En cada nivel se evaluaron cuatro aspectos (pregunta, respuesta, escritura y normas APA) que se puntuaron en una escala de $1 \mathrm{a}$ 5, donde 1 implica el menor nivel de ajuste al criterio. El primer nivel permite producciones con poca profundidad (i.e. textos con ideas periféricas y poco desarrolladas, con opiniones no fundamentadas o solo con citas textuales). El segundo nivel exige procesos de reflexión más elaborados (explicar, argumentar, ejemplificar) sobre conceptos importantes dentro del texto (aunque no sean centrales). En este nivel se exige que la pregunta esté formulada de forma creativa (i.e. crear un caso de análisis en relación con el concepto recuperado). El tercer nivel exige identificar y argumentar ideas y conceptos transversales de los textos (i.e. argumentar la tesis central del autor). El cuarto nivel agrega una exigencia adicional al nivel anterior: vincular las ideas y conceptos centrales entre dos textos.

Antes del inicio de cada nivel se entregó el instructivo correspondiente con la respectiva socialización de los criterios de producción. Posterior a la entrega de sus textos, los estudiantes recibieron retroalimentación grupal tomando como caso de análisis los Bancos de Preguntas más representativos del nivel, tanto los que puntuaron bajo como los que puntuaron alto. Al finalizar el semestre lectivo, los estudiantes fueron exhortados a construir una reflexión escrita sobre su experiencia con el Banco de Preguntas indicando aquellos aspectos que consideraron positivos, negativos, importantes de man- tener, importantes de transformar y aquellos que se debían excluir.

En este estudio exploratorio, de naturaleza cualitativa-descriptiva, se tomaron, con consentimiento informado, las reflexiones escritas de 24 estudiantes de Psicología de VIII semestre (3 hombres y 21 mujeres) en las cuales se evaluaba el Banco de Preguntas a partir de su experiencia subjetiva con la herramienta (33\% de estas reflexiones fueron escritas en 2016 y $67 \%$ en 2017).

El análisis de la información se realizó en dos momentos: organización y categorización. La organización fue elaborada a partir de las mismas consignas que guiaron la reflexión de los estudiantes. Una vez los datos fueron organizados, se establecieron los elementos comunes en las diferentes respuestas facilitando la emergencia de cuatro categorías de análisis: Exigencia, Relación Estudiante-Texto, Pensamiento de Alto Orden y Desafíos al Banco de Preguntas. Estas categorías produjeron una nueva reorganizaron de los datos que permitió someter a discusión las limitaciones y las posibilidades de la herramienta. El análisis de los datos se basa fundamentalmente en el análisis de contenido y utiliza fragmentos de las reflexiones de los estudiantes como parte de la estrategia discursiva utilizada para la reconstrucción del sentido general que se desea presentar respecto de cada categoría. Para mantener la autoría y el anonimato de estos fragmentos, las 24 reflexiones se codificaron con dos letras que aparecen al final de cada enunciado.

\section{Resultados y discusión}

\section{Exigencia}

Una percepción generalizada en los reportes es que el Banco de Preguntas "exige, además de leer el documento, comprenderlo de tal manera que al realizar la pregunta se pueda responder con coherencia" (IC). En esta expresión destaca la división establecida en los procesos de leer y comprender, 
como si la comprensión fuese un aspecto opcional del proceso. Esta división, que podría surgir cuando el estudiante percibe que el texto no le ofrece toda la información necesaria para "comprender", exige acudir a otras fuentes de información, y es justamente esta actividad la que se percibe como una primera exigencia: "te obliga a conocer e indagar para llegar a comprender" (LFC) y exige "un esfuerzo mucho mayor del que está acostumbrado a realizar, porque este exige no solo una lectura rápida, sino el análisis del texto" (MB).

La estructura "extensiva" (Otálora, 2011) del Banco de Preguntas resultó una "estrategia desafiante" (JM) que, ya en el nivel II, "exige a los estudiantes ir más allá de sus capacidades" (JG) e incentiva "usar su creatividad al momento de realizar preguntas en un formato distinto al tradicional" (IC), lo que lleva "a pensar [el texto] también en un nivel práctico" (RC), rompe con la rutina de la lectoescritura y hace el "trabajo menos tedioso" (AG). El último nivel, que solicita relaciones de intertextualidad, resultó "interesante [...] para la comprensión de nuevos conceptos", a propósito de lo cual se argumentó así: "siempre he realizado las lecturas desligado de otros autores que me podrían estar explicando el mismo concepto, pero con un abordaje distinto" (MB).

Es interesante notar que el aumento gradual de las demandas en cada nivel fue percibido en general como un desafío que estaba dentro de las posibilidades de realización de las estudiantes. Esta relación entre el grado de dificultad de la tarea y la positiva autopercepción sobre sus propias habilidades para realizarla es uno de los ingredientes fundamentales de motivación en las actividades de enseñanza-aprendizaje (Shumow y Schmidt, 2014). La motivación en este caso tendría una relación estrecha con la estructura de la tarea; por consiguiente, crear desafíos cognitivos ajustados a la "zona de desarrollo próximo" del estudiante podría ser una estrategia para mantenerlo comprometido con su proceso lectoescritor.
Vale la pena destacar que una estudiante indica que en los niveles superiores del Banco la exigencia de atender a los elementos centrales del texto debería flexibilizarse, de tal manera que permita realizar preguntas-respuestas sobre "otros aspectos expuestos [en el texto] en los que haya tenido mayor comprensión, ya que en ocasiones [dice] se me dificulta llegar con la idea central del autor" (MM). Este reporte podría resaltar el desarrollo del lectoescritor como un proceso de avances y retrocesos (PucheNavarro, 2008) y es coherente con la perspectiva aquí defendida. La afirmación implica investigar si la dificultad enunciada es un tema de reorganización de la estrategia de lectoescritura utilizada por la estudiante o si los cuatro niveles necesitan ser ajustados para capturar pasos intermedios que faciliten operar adecuadamente con dicha exigencia.

\section{Relación estudiante-texto}

El Banco de preguntas ayuda a los "estudiantes abordar los textos de manera distinta y más favorable para el aprendizaje" (MB), "incita a leer más de una vez la lectura" (VM), de tal forma que haya "una revisión exhaustiva y profunda del texto, hasta que se comprende al menos parcialmente" (CM), pues no es "posible elaborar un buen banco de preguntas sin antes haber realizado una lectura profunda y crítica de los textos respectivos, lo cual potencia la habilidad lectora" (PG). Así, esta herramienta "implica entender, reflexionar, criticar, argumentar [acerca del texto] y debatirlo con uno mismo y no se queda simplemente en el hecho de memorizar un apartado o un fragmento de la lectura" (RC).

Estas percepciones refuerzan la idea de que el acto de problematizar un texto y responder a dicho problema genera una relación más activa con el texto (Valera y Madriz, 2002). Las relaciones significativas que algunos estudiantes establecen contrastan con otras relaciones de tipo instrumental que se enfocan más en el resultado que en el proceso de aprendizaje: hacer el Banco influye de manera "personal, pues implica que semanalmente se logre una 
mejor pregunta para una nota mejor" (DT); "muchas ocasiones leí en función de encontrar una pregunta en el texto que fuera apropiada según los criterios de evaluación" (CM); "es posible que este tipo de preguntas se creen con el fin de 'buscar una respuesta', mas no [se dirijan] a hacer una reflexión final acerca de la temática trabajada por los autores" (DL).

Aunque no hay datos que permitan comprender mejor las causas de este tipo de relaciones, es viable considerar un análisis de la pertinencia y complejidad de las lecturas, como también de los niveles y sus exigencias, para identificar cuál elemento de la estrategia facilita la emergencia de estos vínculos. Finalmente, hay consideraciones instrumentales sobre la herramienta que también son positivas para los estudiantes, toda vez que ella "obliga a la lectura de los textos" (PG) y a llegar preparados a clase, siendo "un apoyo a la hora de estudiar para cada parcial" (IO).

\section{Pensamiento de alto orden}

"Pensaba que hacer una pregunta [acerca] de un texto era muy fácil, pero a la hora de construirla se debe analizar lo que se va a preguntar, pues como también se debe dar la respuesta, entonces ahí está lo mejor, que no es hacer una pregunta por hacerla, es también reflexionar" (AG); "aprendí que no es mi opinión, sino la postura del autor y que, así [yo] represente de alguna manera lo que este quiere decir, necesito generar competencias que me permitan conceptualizar sin perder la idea central de cada texto" (MB). En estos fragmentos se observa cómo los estudiantes toman conciencia de sus propios estados de conocimiento y sus transformaciones como lectoescritores. Esta toma de conciencia, según lo reportan Osses y Jaramillo (2008), es un factor clave para el desarrollo del funcionamiento metacognitivo y, por lo tanto, de la habilidad para aprender a aprender, es decir, la capacidad del estudiante para autorregular sus propios procesos de aprendizaje. El Banco de Preguntas parece facilitar en los estudiantes este cuestionamiento, que no solo se limita a la toma de conciencia del conocimiento, sino también a su reorganización, y es esta reorganización del conocimiento, y no únicamente su adquisición, lo que produce desarrollo cognitivo (Karmiloff-Smith, 1994).

Por conocimiento debe entenderse no solo los contenidos semánticos, sino también las estrategias cognitivas que se van desarrollando cuando se resuelven situaciones problema (Karmiloff-Smith, 1994). Un paso clave en la consolidación de estas estrategias se da cuando el sujeto logra desacoplar la estrategia de la situación particular en que fue desarrollada y transferirla a situaciones novedosas. Esto es algo que el Banco de Preguntas parece favorecer: "en un futuro nos servirá en diversas situaciones" (DT); es una "metodología [que] permitió llevar a cabo las diferentes técnicas utilizadas para recolectar información sobre un tema determinado [...]. Como futuros profesionales de la psicología, debemos ser capaces de construir, atribuir valores y reflexionar a partir del significado de lo que leemos en una amplia gama de textos" (ES).

Los relatos presentados refuerzan la idea de que el Banco de Preguntas está fundamentalmente orientado al desarrollo de estrategias de pensamiento de alto orden, entre las cuales destacan el pensamiento abstracto y la metacognición, procesos cognitivos relacionados con el aprendizaje autónomo, la autorregulación y la trasferencia de conocimiento a situaciones novedosas (Osses y Jaramillo, 2008).

\section{Desafíos al Banco de Preguntas}

Desde la perspectiva de los estudiantes, hay aspectos que deben ser discutidos y mejorados. Aunque la inclusión del criterio de "creatividad" en el nivel II supuso un desafío para unos, para otros el propio criterio resulta ambiguo y supone un riesgo: "que por cumplir con el criterio de creatividad se perdía el sentido general o la coherencia en relación directa con la idea central del texto" (VP). Aquí vale la pena señalar que el nivel II del Banco es el primer momento en el que los estudiantes deben comprender y hacer uso flexible del conocimiento (Stone, 
1999), de tal manera que puedan organizar sus preguntas-respuestas más allá del formato tradicional básico de enunciación, por ejemplo, inventando un caso o situación para analizar un concepto. Debido a lo anterior, quizás sea pertinente refinar el instrumento para facilitar el paso a estas formas de pensamiento abstracto.

No cabe duda de que la claridad en la instrucción y los criterios de evaluación en cada nivel son fundamentales para la resolución del Banco de Preguntas; sin embargo, la instrucción por sí misma podría ser insuficiente. Varios estudiantes que no reportan problemas con las instrucciones sugieren que el docente realice un primer Banco en la primera clase, con el fin de modelar la ejecución. Este requerimiento muestra que, en las fases iniciales del aprendizaje de una habilidad, aplicar un modelo de aprendizaje social (Bandura, 1984) podría tener mejores efectos en el desempeño a largo plazo.

Un segundo aspecto a considerar es el efecto en las relaciones interindividuales. Como estrategia de preparación para la clase, el Banco de Preguntas hace que esta "sea más dinámica y únicamente no esté a cargo del profesor, sino más bien que cada uno aporte desde su punto de vista según lo que plantea las lecturas" (MM). Este aspecto dinámico e interactivo contrasta con la emergencia de otras formas de relación, más competitivas e instrumentales, en las que "cada quien cumple con su pregunta" (RC) trabajando de forma aislada sin acudir al diálogo entre pares.

Según Aguirre (2015), las prácticas educativas universitarias, en un mundo altamente competitivo y meritocrático como el actual, configuran dispositivos de subjetivación (Larrosa, 2000) donde el individualismo y la indiferencia aparecen como formas privilegiadas del ser para la supervivencia del sujeto. Dirigir la observación del estudiante a la lógica de funcionamiento social exige tomar una posición política frente al acto educativo, para que este vehicule no solo la apropiación de contenidos y estrategias cognitivas, sino también las formas de resistencia social a las lógicas socioeconómicas dominantes (Giroux, 1985). Un ejemplo de esta resistencia social lo discute y propone Ovejero (2012b) a través del aprendizaje cooperativo, una forma de trabajo escolar que recrea y fortalece la idea del trabajo comunitario como estrategia efectiva para afrontar la fragmentación social y sus efectos.

El tercer aspecto a considerar tiene que ver con la retroalimentación. Algunos estudiantes consideran que deberían implementarse estrategias de retroalimentación diversificadas para mejorar el desempeño en la lectoescritura de los Bancos, como, por ejemplo, la entrega posterior a la clase y no antes (DT) o la retroalimentación personalizada verbal o escrita (IC). Estas sugerencias están en sintonía con la heterogeneidad del cuerpo estudiantil y abordarlas supone un desafío académico-administrativo fundamental para asegurar la calidad de la formación, pues la retroalimentación sistemática, además de sustentar el proceso de aprendizaje, le permite al sujeto ir construyendo estrategias de autorregulación basadas en criterios públicos y explícitos (Stone, 1999).

\section{Discusión}

En este artículo se aborda el tema de las (in)competencias de los estudiantes en educación superior como un problema localizado en las prácticas educativas universitarias. Buscar la causa del bajo desempeño académico en déficits de orden biológico, psicológico o familiar acarrea importantes repercusiones políticas y socioeconómicas en los sujetos escolarizados (Baquero, 2009), pues determina categorías como el "estudiante problema" y procedimientos como la "educación especial" (Ovejero, 2012a; Brunner, 2010; Giroux, 1985). Estas prácticas y categorías terminan por fabricar justamente aquello que dicen intervenir: al sujeto con necesidades especiales (Larrosa, 2000) y, por consiguiente, terminan por instalar las condiciones necesarias para producir procesos psicológicos igualmente deficitarios (Baquero, 2009). 
Sin negar el papel que cumplen las variables individuales en el desarrollo del lectoescritor competente, el análisis aquí se enfoca en explorar cómo una estrategia utilizada de forma cotidiana por los docentes se puede transformar en una herramienta que fortalece de forma cuidadosa y sistemática competencias en lectura y escritura que, debido al origen social y cultural de los estudiantes, podrían no haber sido objeto de desarrollo. Al ser una herramienta intensiva y extensiva (Otálora, 2011), el Banco de Preguntas alcanza diferentes niveles de complejidad que parten de las competencias reales del estudiante y lo llevan a desempeños cada vez más expertos. Esta característica parece haber tenido efectos positivos en la motivación de las estudiantes, quienes en su mayoría asumieron la complejidad cambiante como un reto interesante. Esto muestra que la motivación en clase no es un sinónimo de entretenimiento, sino del adecuado balance entre el desafío cognitivo propuesto y las habilidades disponibles para superarlo (Shumow y Schmidt, 2014).

En cualquiera de sus niveles, el Banco de Preguntas parece exigir la metacognición como estrategia cognitiva central, proceso fundamental en la habilidad para aprender a aprender (Osses y Jaramillo, 2008). Excepto el primer nivel, la descontextualización (Baquero, 2009) es una exigencia cognitiva que el Banco de Preguntas requiere para el planteamiento de preguntas-respuestas, operación mental que implica identificar ciertas características relevantes de una situación (un concepto del texto, por ejemplo), aislarla de la situación (abstracción) y transferirla a un nuevo contexto de aplicación (recontextualización), lo cual es justamente lo que se espera del estudiante con formación universitaria. Los impactos de la herramienta, en esta perspectiva, se extienden más allá de los límites del lectoescritor competente.

Luego de trabajar los textos a través del Banco de Preguntas, algunos estudiantes reportan percibirse como lectores más críticos, sin que expliquen en profundidad lo que eso significa. Por la forma en la que están construidos los distintos niveles, el estudiante debe no solo enunciar respuestas, sino explicarlas, argumentarlas, ejemplificarlas y/o relacionarlas con otros textos. Es posible que estas características de la herramienta, interiorizadas por el estudiante, sean lo que define parte de su autopercepción como lector crítico.

Lo anterior fortalece la idea de que los instrumentos de mediación semiótica, más que facilitar el desarrollo de ciertas capacidades, establecen las condiciones para su fabricación (Larrosa, 2000; Baquero, 2009). No obstante, los estudiantes podrían tener una conciencia limitada de sus propios estados del conocimiento (Silvestri, 2006), con lo cual el cambio real solo puede rastrearse de forma objetiva en un estudio longitudinal que analice las posibles trasformaciones en la producción escrita de los estudiantes (Tovar y Aristizábal, 2019) y que responda a algunas cuestiones de interés: ¿hay realmente un cambio que subyace al uso de la herramienta? Si lo hay, ¿cuál es la naturaleza y magnitud del cambio? ¿Cuál sería el impacto de la herramienta en la formación profesional si, en vez de ser utilizada en estudiantes de semestres avanzados, fuera utilizada con estudiantes de primeros semestres? ¿Cuáles serían las consideraciones a tomar?

\section{Conclusión}

A través de sus años de formación profesional, todos los estudiantes adquieren cierto tipo de habilidad lectoescritora que no necesariamente coincide con lo que la universidad espera desarrollar en sus estudiantes. Si esa adquisición se da en un contexto de enseñanza-aprendizaje explícito, organizado, sistemático, comunitario y dentro de las propias disciplinas, más que como proceso empírico, accidental, aislado o como una actividad extracurricular descontextualizada, es posible que los educandos puedan obtener mayor beneficio en sus diversas asignaturas.

El establecimiento de esos contextos exige, sin embargo, un coste que debe ser sopesado por do- 
centes e instituciones, pues requiere promover el desarrollo de una habilidad que, se presupone, no está madura al ingresar a la educación superior y que tradicionalmente no se considera objeto de enseñanza (Carlino, 2001). El fortalecimiento de dicha habilidad se puede hacer a través de ciertos ajustes en las actividades cotidianas de lectura y escritura que el docente desarrolla en su clase (como es el caso del Banco de Preguntas), pero requiere estar soportado por políticas institucionales que incluyan la lectoescritura como un asunto curricular (Carlino, 2001). De esta manera, el desarrollo del lectoescritor competente no se queda en la iniciativa personal y aislada de los docentes, sino que se convierte en una responsabilidad de toda la comunidad académica.

Para finalizar, la competencia lectoescritora, si bien es importante, no asegura por sí sola el éxito académico del cuerpo estudiantil. Existen habilidades de otra naturaleza, igualmente importantes, que la academia ha de desarrollar; por lo tanto, el Banco de Preguntas hace un aporte a aquellas habilidades y conocimientos que requieren un lectoescritor activo que puede regular algunos de sus procesos de comprensión y producción textual y sostener un diálogo académico con sus pares y profesores a partir de un cuestionamiento personal.

\section{Referencias}

Aguirre, A. (2015). A Relação Professor-Estudante na Univerisdade Pública. Uma Leitura Bakhtiniana (Tesis de maestría). Universidad Federal de Paraná, Curitiba-Brasil.

Bajtín, M. (1997). Marxismo e filosofía da linguagem. Sao Pablo: Ucitec.

Bandura, A. (1984). Teoría del aprendizaje social. Madrid: Espasa-Calpe.

Baquero, R. (2009). Desarrollo psicológico y escolarización en los enfoques socioculturales: nuevos sentidos de un viejo problema. Avances en Psicología Latinoamericana, 27(2), 263-28o. Recuperado de http://www. scielo.org.co/pdf/apl/v27n2/v27n2a5.pdf

Brunner, J. (2010). Lenguaje del hogar, capital cultural y escuela. Pensamiento Educativo, 46(1), 17-44. Recuperado de https://pensamientoeducativo.uc.cl/index.php/pel/article/view/459

Carlino, P. (2001). Hacerse cargo de la lectura y la escritura en la enseñanza universitaria de las ciencias sociales y humanas. I Jornadas sobre la Lectura y la Escritura como Prácticas Académicas Universitarias. Departamento de Educación, Universidad Nacional de Luján, Luján, Provincia de Buenos Aires.

Carlino, P. (2007). ¿Qué nos dicen las investigaciones internacionales sobre escritura en la universidad? Cuadernos de Psicopedagogía, 4, 21-40. Recuperado de https://revistas.uptc.edu.co/index.php/psicopedagogia/ article/view/505

Collante, C. (2010). El valor de la pregunta. Revista Investigación en Marcha, 3, 21-27. Recuperado de http://app. infotephvg.edu.co/cienaga/hermesoft/portal/home_1/rec/arc_811.pdf

Cubero, R. (2005). Elementos básicos para un constructivismo social. Avances en Psicología Latinoamericana, 23, 43-61. Recuperado de https://revistas.urosario.edu.co/index.php/apl/article/view/1240 
Estienne, V.y Carlino, P. (2004). Leer en la universidad: enseñar y aprender una cultura nueva. Unipluriversidad, 4(3), 9-17. Recuperado de http://aprendeenlinea.udea.edu.co/revistas/index.php/unip/article/viewArticle/12210

Faraco, C. (2003). Linguagem e diálogo: as ideias linguisticas do círculo de Bakhtin. Curitiba: CRIAR.

Giroux, H. (1985). Teorías de la reproducción y la resistencia en la nueva sociología de la educación: un análisis crítico. Cuadernos Políticos, 44, 36-65. Recuperado de http://www.cuadernospoliticos.unam.mx/cuadernos/contenido/CP.44/cp.44.6.\%20HenryAGiroux.pdf

Karmiloff-Smith, A. (1994). El desarrollo tomado en serio. En J. Gómez y M. Núñez (eds.), Más allá de la modularidad (pp. 17-49). Madrid: Alianza.

Larrosa, J. (2000). Tecnologias do Eu e Educação. En T. Silva (org.), O Sujeito da Educação (pp. 35-86). Petropolis: Vozes.

Ochoa, S. y Aragón, L. (2007). Funcionamiento metacognitivo de estudiantes universitarios durante la escritura de reseñas analíticas. Universitas Psychologica, 6(3), 493-506. Recuperado de https://revistas.javeriana. edu.co/index.php/revPsy cho/article/view/135

Olave, G., Cisneros, M. y Rojas, I. (2013). Deserción universitaria y alfabetización académica. Educación y Educadores, 16, 455-471. Recuperado de http://educacionyeducadores.unisabana.edu.co/index.php/eye/article/ view/2726/

Ortiz, E. (2011). La escritura académica universitaria: estado del arte. Íkala, 16(28), 17-41. Recuperado de http:// www.scielo.org.co/scielo.php?script=sci_abstract\&pid=S0123-34322011000200002

Osses, S. y Jaramilo, S. (2008). Metacognición: un camino para aprender a aprender. Estudios Pedagógicos, 34(1), 187-197. DOI: 10.4067/S0718-07052008000100011

Otálora, Y. (2011). Diseño de espacios educativos significativos para el desarrollo de competencias en la infancia. CS, 5, 71-96. DOI: $10.18046 /$ recs.i5.452

Ovejero, A. (2012a). El fracaso escolar. Un fenómeno construido socialmente. En A. Hincapié y M. Morales (comps.), Subjetividad, memoria y educación. Contextos de aplicación de la psicología social en un mundo en crisis (pp. 323-350). Medellín: Universidad Pontificia Bolivariana.

Ovejero, A. (2012b). El aprendizaje cooperativo como vía de solución de los principales problemas escolares. En A. Hincapié y M. Morales (comps.), Subjetividad, memoria y educación. Contextos de aplicación de la psicología social en un mundo en crisis (pp. 383-410). Medellín: Universidad Pontificia Bolivariana.

Perinat, A. (1995). Los procesos recursivos en la mente humana. Revisión de "Prolegómenos para una teoría del juego y del símbolo". Cognitiva, 7(2), 59-126. Recuperado de https://dialnet.unirioja.es/servlet/ articulo?codigo $=122634$ 
ISSN 0123-1294 | e-ISSN 2027-5358 | Educ.Educ. Vol. 22. No.1 | Enero-abril de 2019 | pp. 67-80.

Universidad de La Sabana | Facultad de Educación

Puche-Navarro, R. (2008). Érase una vez el desarrollo. En J. Larreamendy-Joerns, R. Puche-Navarro y A. Restrepo Ibiza (comps.), Claves para pensar el cambio: ensayos sobre psicología del desarrollo (pp. 71-94). Bogotá: Uniandes.

Rosas, R.y Balmaceda, C. (2008). Solistas. En Piaget, Vigotski y Maturana: constructivismo a tres voces (pp.11-78). Buenos Aires: Aique.

Shumow, L.y Schmidt, J. (2014). Enhancing Adolescents Motivation for Science: Research-Based Strategies for Teaching Male and Female Students. Thousand Oaks, CA: Corwin.

Silvestri, A. (2006). La formulación de preguntas para la formulación de textos. Revista Signos, 39(62), 493-510. DOI: 10.4067/S0718-0934200600030000

Stone, M. (1999). ¿Qué es la enseñanza para la comprensión? En M. Stone (comp.), La enseñanza para la comprensión (pp. 95-126). Buenos Aires: Paidós.

Tovar, A. y Aristizábal, D. (2019). Instrumentos cotidianos para el fortalecimiento de la lectoescritura en educación superior: efectos del Banco de Preguntas. Documento en construcción.

Valera, G. y Madriz, G. (2002). Las preguntas en las ciencias humanas. Un estudio ecológico de aula universitaria. Revista Investigación en la Escuela, 48, 81-94. Recuperado de http://hdl.handle.net/11441/60605

Vigotsky, L. (1988). El desarrollo de los procesos psicológicos superiores. México: Grijalbo [Mind in Sociely: The development of higher psychological processes. Cambridge: Harvard University Press]. 